\title{
Miniaturized humidity and temperature sensing RFID enabled tags
}

\author{
Javeria Anum Satti ${ }^{1} \quad$ | Ayesha Habib ${ }^{1}$ | Hafsa Anam ${ }^{1}$ | Sumra Zeb1 \\ Yasar Amin ${ }^{1,2}$ | Jonathan Loo ${ }^{3}$ | Hannu Tenhunen ${ }^{2,4}$ \\ ${ }^{1}$ ACTSENA Research Group, Department of Telecommunication Engineering, University of Engineering and Technology, Taxila, Punjab 47050, Pakistan \\ ${ }^{2}$ iPack Vinn Excellence Center, Department of Electronic Systems, Royal Institute of Technology (KTH), Isafjordsgatn 39, Stockholm SE-16440, Sweden \\ ${ }^{3}$ Department of Computer Science, School of Engineering and Information Sciences, Middlesex University, London, United Kingdom \\ ${ }^{4}$ Department of Information Technology, TUCS, University of Turku, Turku 20520, Finland
}

\begin{abstract}
A compact 27-bit linearly polarized chipless radio frequency identification tag is presented in this research. The proposed tag is designed with an overall tag dimension of $23 \times 23 \mathrm{~mm}^{2}$. The tag comprises of metallic (copper) rings-based structure loaded with slots. These slots correspond to a particular sequence of bits. The circular tag is analysed using 2 different substrates, that is, Rogers RT/duroid/5870 and flexible Rogers RT/duroid/5880. The radar cross-section response of frequency signatured tag is analysed for humidity and temperature sensor designs. Humidity sensing is achieved by deploying a DuPont Kapton HN heat resistant sheet on the shortest slot of the tag, that is, the sensing slot. Temperature sensing is attained using Rogers RT/ duroid/5870 and Stanyl polyamide as a combined substrate. Hence, the miniaturized, robust, and flexible tag can be deployed over irregular surfaces for sensing purposes.
\end{abstract}

\section{K E Y W O R D S}

chipless tag, electromagnetic, flexibility, humidity sensor, temperature sensor

\section{1 | INTRODUCTION}

Internet of things (IoT) also known as ambient intelligence is an emerging technology. It ensures connectivity of physical and virtual objects to the internet at an unprecedented rate, thus, transforming traditional objects into smart ones. ${ }^{1,2}$ This technology is an integral part of internet future, and it will interconnect uniquely identified embedded items through ubiquitous communication of physical objects. ${ }^{3,4}$ IoT uses wireless and pervasive computing technologies to make objects intelligent. ${ }^{1,5}$

The idea of IoT can be implemented using radio frequency identification (RFID) technology. RFID is a promising technology which has revolutionized automatic identification and wireless sensing of tagged objects. ${ }^{6} \mathrm{RF}$ waves are exchanged between a tag and reader using wireless transmission. RFID is a fairly handy technology because it facilitates non-line of sight communication which improves the tag's efficiency and provides longer reading range. ${ }^{7-9}$ At the item level tracking, RFID technology is replacing optical barcodes. ${ }^{7}$

The fabrication of microchip circuits is really expensive, and this sets a certain limitation on the manufacturing of RFID tags. This problem can be solved by excluding the microchip from the tag design. RFID tag without silicon chip is famous as chipless RFID tag. ${ }^{10-12}$ Chipless tags have already gained attention due to the removal of microchips from the circuitry and are aimed to further drive the costs down using techniques such as 'inkjet printing'. Other than low-cost expectations, these tags ensure stronger robustness, long read range and advanced real-time performance, 
avoiding delays. ${ }^{13}$ In chipless RFID tags, an integrated circuit (IC) and self-empowerment are not required; hence, these tags do not utilize transmission protocols for detection. ${ }^{14}$ The data are encoded using electromagnetic (EM) waves instead of the ICs (which are expensive). The tag is excited by EM waves, and the encoded backscattered signal is received by the reader. The tag responses can be analysed in the time domain or the most commonly used frequency domain. ${ }^{15,16}$

The chipless RFID tags are further examined to make the tag capable of performing sensor functions. ${ }^{17}$ Various researches have been made in the field of chipless RFID tags with integrated sensors. In Ref. 18, a poly-vinyl alcohol (PVA)-based humidity sensor is presented. In this article, the $\mathrm{RH}$ sensing is first done using PVA dielectric film and in addition to this, the sensing behaviour is also realized using Kapton HN polymer. A comparative analysis is drawn between the 2 techniques.

In Ref. 19, a 30-bit tag, incorporating an additional feature of temperature sensing is presented. In this work, Stanyl polyamide resin is used for temperature sensing. The Stanyl polymer is placed beneath the 2 innermost slots of the tag, which ultimately detects the temperature change.

This research article presents a passive, high capacity, chipless RFID tag. The 27-bit circular shaped tag with openended slots is capable of storing a large amount of data while remaining in a compact tag size of $23 \times 23 \mathrm{~mm}^{2}$. The metal portion in the tag design corresponds to the capacitive part, whereas, the slots represent the inductive element. The combination of binary numbers is generated using slot/resonator structure. Each slot is responsible for yielding ' 1 ' bit and the metal portion generates ' 0 ' bit. Hence, in this way, the data are encoded in the proposed tag.

The linearly polarized tag design is realized for rigid (Rogers RT/duroid/5870) as well as flexible (Rogers RT/ duroid/5880) substrate. The frequency band for Rogers RT/ duroid/5870 is $3.3-13.5 \mathrm{GHz}$, whereas, the operational spectrum for flexible Rogers RT/duroid/5880 is $3.5-14.0 \mathrm{GHz}$. The flexible characteristic of the tag makes it suitable to be deployed on a variety of objects, for example, the irregular curvatures of bottles.

Furthermore, the effectiveness of the tag is enhanced by adding the sensing attribute. Humidity sensing trait is attained by deploying DuPont Kapton HN heat resistant tape on the shortest slot that corresponds to the least significant bit (LSB). The feature of temperature sensing is achieved with the aid of polyamide resin that is temperature dependent. Hence, data dense, finite- sized, flexible, sensor integrated, the low-cost tag is proposed. The novelty of the proposed tag design relies on high data storage capacity, flexibility, and sensor integration in smaller tag size as compared to the already proposed work as shown in Table 1.
The basic arrangement of the research article is as follows: section 2 introduces the working principle of the tag. Section 3 includes the structure and relative dimensions. Section 4 is the major section with 2 subsections. It starts with the discussion of the results achieved. The first part explains humidity sensing while the other one covers the temperature sensing. The proposed research article is concluded in section 5.

\section{2 | WORKING PRINCIPLE}

The basic working principle of chipless RFID tag is based on backscattering phenomena; similar to that used by conventional pulsed radars. The backscattering operation is shown in Figure $1^{22}$ which consists of a reader also known as 'interrogator' and a chipless RFID tag additionally known as 'transponder'.

The reader is used to activate and hear the tag. It basically consists of 2 main components which include a vector network analyser (VNA) and the antenna subsystem. VNA performs 2 core functions, that is, generation of the signals to be transmitted and the detection of the signals that are reflected back towards the reader. Antenna subsystem is used for the production of the polarized signals. It also performs the functions of transmitting and receiving of the signals. ${ }^{23}$ The reader transmits an EM wave. If the chipless tag is present in the vicinity of the reader, it absorbs the transmitted EM waves which stimulate a current on the metallic surface of the tag. As a result, a modulated wave is generated that is scattered back towards the reader. This wave is known as backscattered signal, which contains the encoded data. ${ }^{24}$ The unique tag's ID is used for the identification of the received information.

The radar cross-section (RCS) response of the tag is measured at Fraunhofer far-field distance that can be calculated by Equation 1.

$$
R=\frac{2 D^{2}}{\lambda}
$$

Here, $R$ is the far-field distance, $D$ is the largest dimension of the tag, and $\lambda$ is the wavelength. ${ }^{25}$

The receiving antenna collects the power that is broadcasted by the transmitting antenna. This transmitted power is given by Friis transmission equation, ${ }^{26}$

$$
\frac{P_{\mathrm{RX}}}{P_{\mathrm{TX}}}=\left(\frac{\lambda}{4 r \pi}\right)^{2} G_{\mathrm{TX}} G_{\mathrm{RX}}
$$

Where the distance between transmitter and receiver is referred as $r, G_{\mathrm{TX}}$ is the gain of the transmitter antenna and $G_{\mathrm{RX}}$ is the gain of receiver antenna, $\lambda$ is the wavelength, 
TABLE 1 Comparison with other chipless RFID tags

\begin{tabular}{|c|c|c|c|c|c|}
\hline $\begin{array}{l}\text { Resonator } \\
\text { shape }\end{array}$ & $\begin{array}{l}\text { Circular } \\
\text { (proposed } \\
\text { research) }\end{array}$ & U-shaped ${ }^{6}$ & Circular $^{20}$ & Hexagonal $^{21}$ & Rectangular $^{7}$ \\
\hline Tag Size $\left(\mathrm{mm}^{2}\right)$ & $23 \times 23$ & $26 \times 70$ & 153.8 & $23 \times 10$ & $15 \times 6.8$ \\
\hline No. of bits & 27 & 20 & 9 & 14 & 6 \\
\hline $\begin{array}{l}\text { Bit density } \\
\left(\mathrm{bit} / \mathrm{cm}^{2}\right)\end{array}$ & 5.1 & 1.09 & 5.8 & 6.08 & 5.8 \\
\hline Flexibility & $\checkmark$ & $\mathrm{x}$ & $\checkmark$ & $\mathrm{x}$ & $\checkmark$ \\
\hline Sensing & $\checkmark$ & $\mathrm{x}$ & $\checkmark$ & $\mathrm{x}$ & $\checkmark$ \\
\hline RCS max (dBsm) & $-23 \sim-35$ & $-31 \sim-40$ & $-6 \sim-14$ & $-25 \sim-31$ & $-29 \sim-31$ \\
\hline
\end{tabular}

$P_{\mathrm{TX}}$ and $P_{\mathrm{RX}}$ are the transmitted and received power, respectively.

The chipless RFID tags can be excited by a linearly or dual polarized EM waves. The incident plane wave is mathematically given as,

$$
\mathbf{E}(x, y, z, t)=E_{o} \exp [j(\omega t-k z)] \hat{\mathbf{x}}+E_{o} \exp \left[j\left(\omega t-k z+\frac{\pi}{2}\right)\right] \hat{\mathbf{y}}
$$

where $\boldsymbol{E}$ is an electric field, $\omega$ is angular frequency, $k$ is wave vector, $t$ represents time, and, $(x, y, z)$ is position vector.

The chipless RFID tag, presented in this research article, is excited by a linearly polarized incident plane wave. The tag is composed of slot/resonator structure where each slot generates ' 1 ' bit. Hence, in this way multiple bits can be accumulated within the tag's structure without the need of an application specific integrated circuit. $^{27}$

\section{3 | CHIPLESS RFID TAG DESIGN}

A compact, highly dense chipless RFID tag is presented in Figure 2. The tag structure resides in a miniature footprint of $23 \times$ $23 \mathrm{~mm}^{2}$ and the total diameter of the circular patch is $21 \mathrm{~mm}$.

The proposed tag is realized on 2 different substrates, that is, Rogers RT/duroid/5870 having a thickness of $0.78 \mathrm{~mm}\left(\varepsilon_{\mathrm{r}}=2.2\right)$ and Rogers RT/duroid/5880 flexible with a thickness of $0.508 \mathrm{~mm}\left(\varepsilon_{\mathrm{r}}=2.2\right)$. The radiator is designed using a copper cladding having a thickness of $35 \mu \mathrm{m}$. The circular shaped design is composed of an arrangement of slots/resonators where each slot corresponds to a bit. The length and width of each slot are varying to make it resonant at a different frequency. Hence, there are 27 slots corresponding to 27 bits generating $2^{27}=134217728$ exclusive Auto-IDs having a bit density of $5.1 \mathrm{bit} / \mathrm{cm}^{2}$. The widths of the slots are given in Table 2.

A circle of diameter $10.8 \mathrm{~mm}$ is designed in the centre of the tag to achieve sharper resonances. According to the
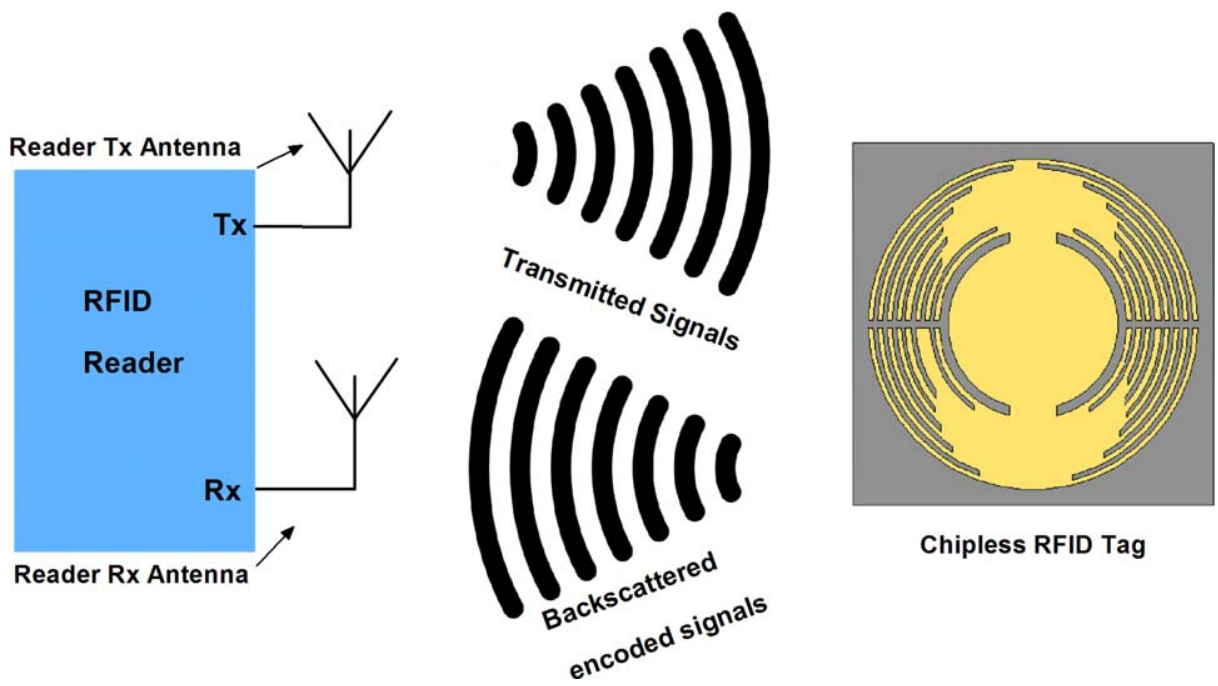

FIGURE 1 Backscattering principle 


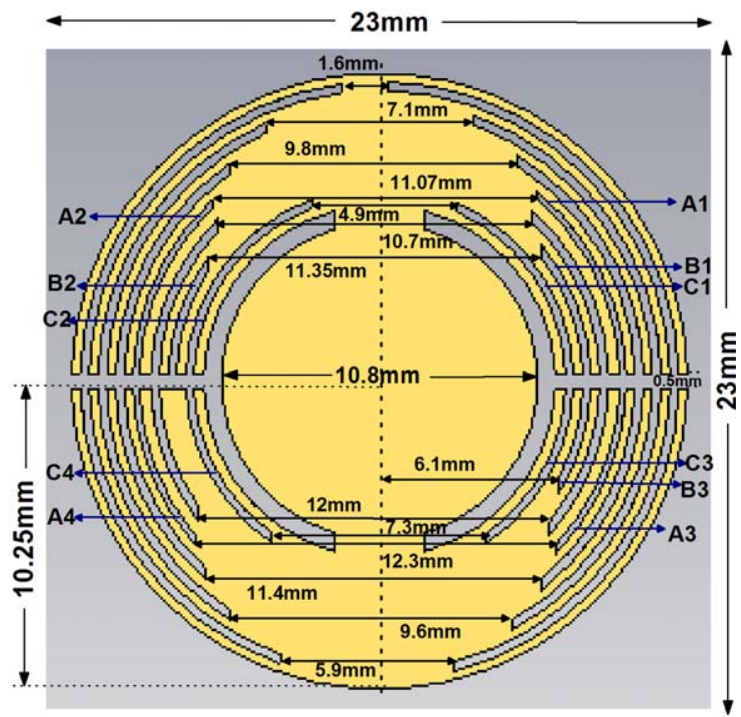

F IGURE 2 Twenty-seven-bit chipless RFID tag design

operation of chipless RFID tags, the longest slot provides the dip at the lowest frequency and is known as a most significant bit while the shortest slot identified as a LSB gives the resonance at the highest frequency. The varying lengths and widths of the slots allow the resonances to be produced at different frequencies.

The resonating frequency of the slot of a particular radius can be determined by the mathematical relation given below,

$$
f_{\text {res }}=\frac{c}{2 \pi R_{\text {slot }}} \sqrt{\frac{2}{\varepsilon_{\mathrm{r}}+1}}
$$

Here, $R_{\text {slot }}$ represents the radius of the slot, $\varepsilon_{\mathrm{r}}$ shows the relative permittivity of the substrate used, and $f_{\text {res }}$ is the resonating frequency of the slot. ${ }^{25}$ Thus, the above-given equation shows that a distinct slot, resonating at a particular frequency relies on the radius of that slot and the relative permittivity of the substrate.

The designing, simulation, and optimization of the introduced tag are performed on CST Microwave Studio Suite. Incident plane wave is used for exciting the tag.

\section{4 | RESULTS AND DISCUSSION}

The tag proposed in this work is capable of generating $2^{27}$ unique bit sequences in a small size. The fabricated tag is scrutinized in a real environment to achieve measured results.

The measurement setup is same as that in Ref. 28, utilizing a combination of transmitting and receiving horn antennas, VNA model R\&SZVL13 and the prototype of the tag under test. In the proposed research, the far-field distance is taken as $30.2 \mathrm{~mm}$ at which the tag is placed to measure the RCS response.

The 27-bit tag, representing a bit word ' 1111111111111 11111111111111', presented in this research article, is
TABLE 2 Widths of the slots

\begin{tabular}{ll} 
Slot name & Slot width $(\mathbf{m m})$ \\
\hline A1-A4 & 0.21 \\
\hline B1-B3 & 0.27 \\
\hline C1-C4 & 0.26 \\
\hline All other slots & 0.3 \\
\hline
\end{tabular}

optimized for 2 different substrates. Figure 3A shows the RCS response of the tag analysed on Rogers RT/duroid/5870 substrate and is named as 'Tag-A'. The results for 'Tag-B' are shown in Figure 3B in which copper radiator is etched on Rogers RT/duroid/5880 flexible substrate. The characteristic comparison of 2 tags, that is, Tag-A and Tag-B is presented in Table 3.

Tag-A covers the frequency band 3.3-13.5 GHz, whereas the total bandwidth of the tag is $10.2 \mathrm{GHz}$.

The RF range for Tag-B, that is analysed using Rogers RT/ duroid/5880 flexible substrate, is $3.5-14.0 \mathrm{GHz}$ and the overall

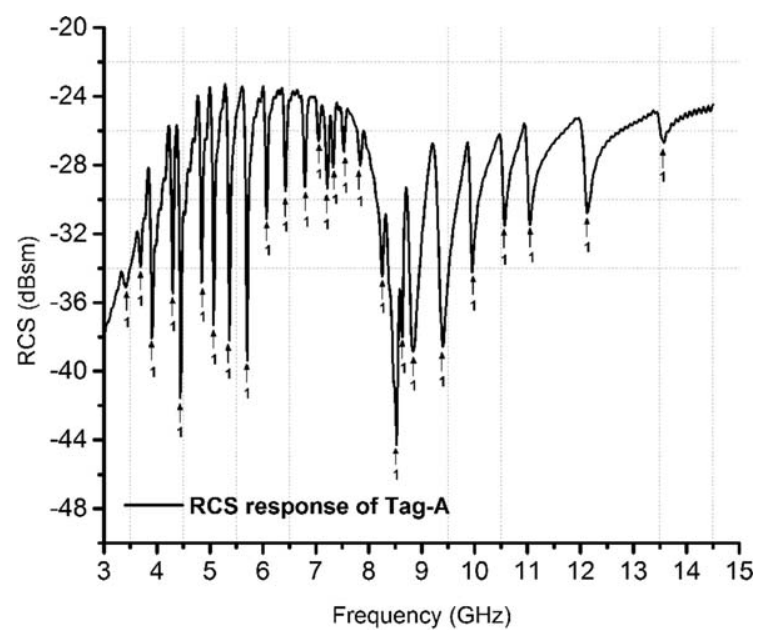

(A)

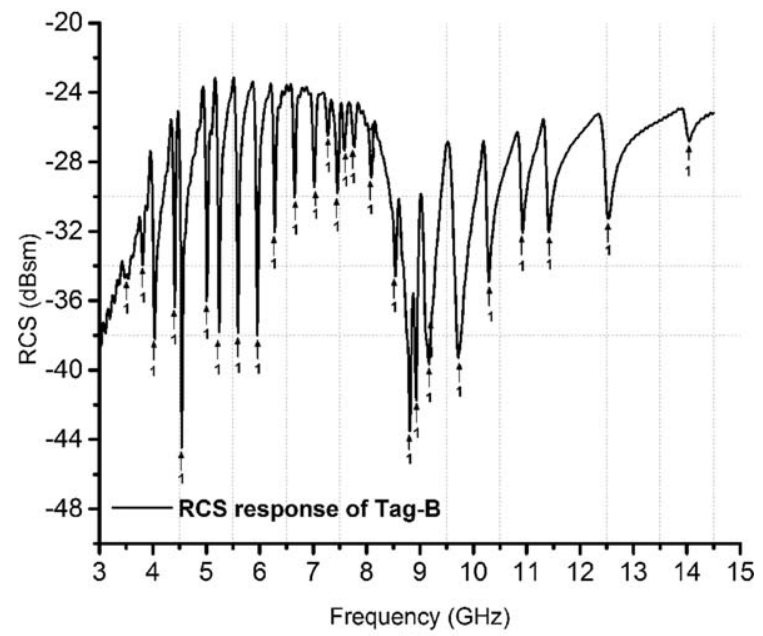

(B)

FIGURE 3 A, RCS response of Tag-A. B, RCS response of Tag-B 
TABLE 3 Comparative study of Tag-A and Tag-B

\begin{tabular}{|lll|}
\hline Characteristics & Tag-A & Tag-B \\
\hline Substrate & $\begin{array}{l}\text { Rogers RT/ } \\
\text { duroid } / 5870\end{array}$ & $\begin{array}{l}\text { Rogers RT/ } \\
\text { duroid } / 5880\end{array}$ \\
\hline Thickness $(\mathrm{mm})$ & 0.78 & 0.508 \\
\hline Permittivity & 2.2 & 2.2 \\
\hline Loss tangent & 0.0009 & 0.0009 \\
\hline Radiator & Copper & Copper \\
\hline Bandwidth $(\mathrm{GHz})$ & 10.2 & 10.5 \\
\hline Freq. band $(\mathrm{GHz})$ & $3.3-13.5$ & $3.5-14.0$ \\
\hline Flexibility & - & $\checkmark$ \\
\hline
\end{tabular}

bandwidth is $10.5 \mathrm{GHz}$. This shows that the displacement of the substrate from rigid to flexible shifts the frequency band towards the right and the operational bandwidth increases.

Figure 4 shows the measured and computed results of Tag-A along with its prototype. It can be seen that the measured and computed response of the tag displays an acceptable agreement. A slight drift along the frequency axis is observed for some resonant dips which are easily detectable with the reader setup.

The surface induced current patterns at 2 different frequencies, using Rogers RT/duroid/5870 substrate is depicted in Figure 5. The Figure 5A shows the surface current distribution at the lowest frequency, whereas, the Figure 5B presents the mapping of the induced current at the highest frequency.

Each slot gives 1 dip on a distinct resonant frequency. When the tag is excited with an incident plane wave, a particular tag ID is generated. Each slot in the structure corresponds to a logic state ' 1 '. For shifting the logic state from ' 1 ' to ' 0 ', the slot can either be shorted or removed completely. Hence, the shorted or removed slot will not give any dip at a particular resonant frequency and will correspond to

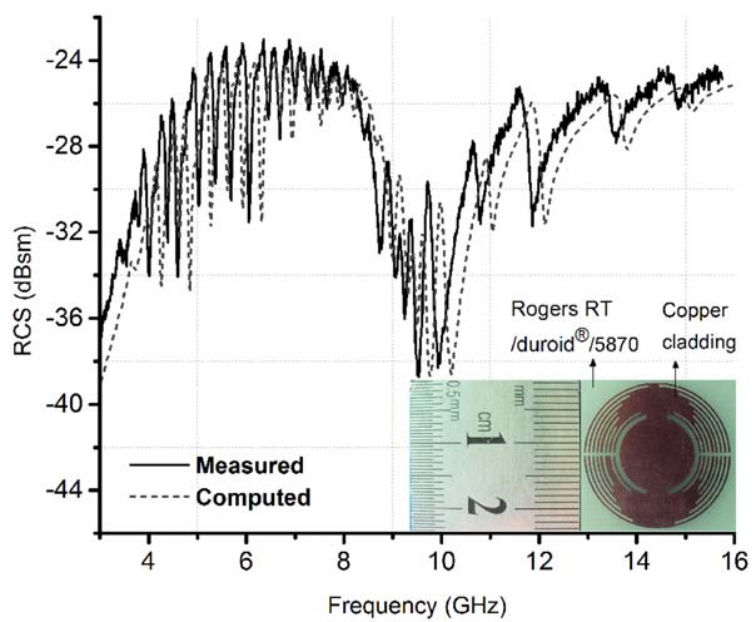

FIG URE 4 Measured and computed RCS response of Tag-A

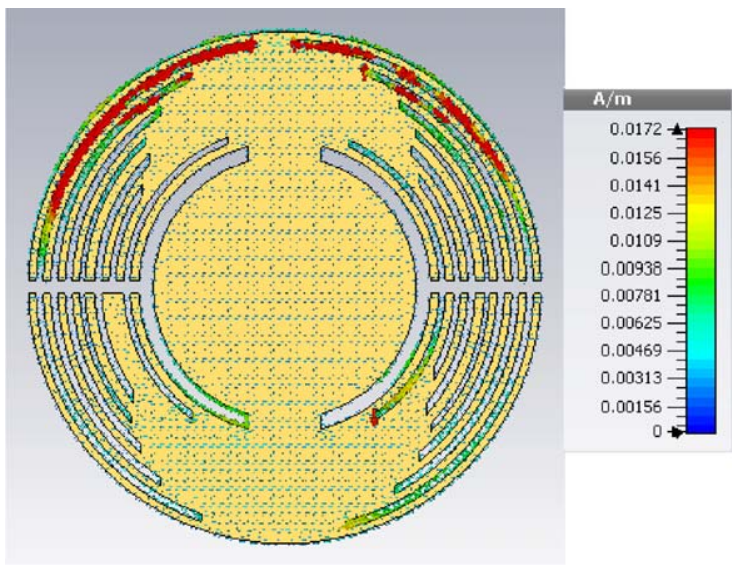

(A)

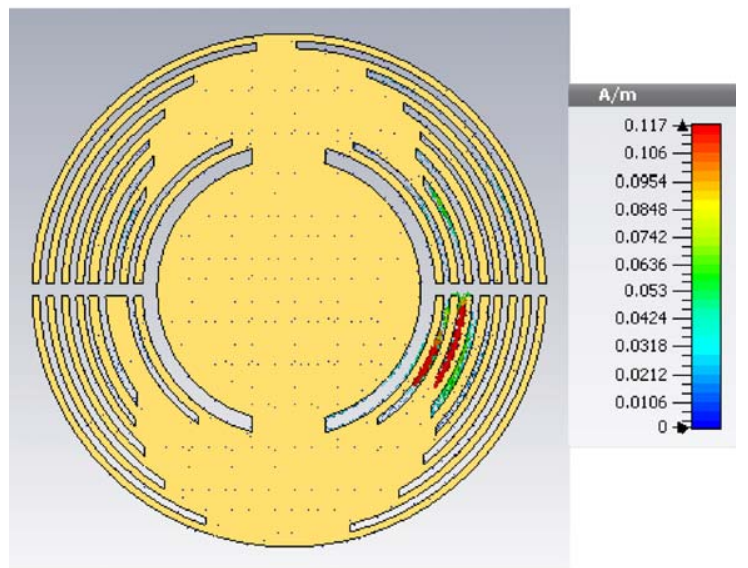

(B)

FIG URE 5 A, Current distribution at lowest frequency (3.3 GHz). $\mathrm{B}$, Current distribution at highest frequency $(13.5 \mathrm{GHz})$

'0' logic state. Every slot has a different frequency response; hence, shorting any slot will have a very little effect on the frequency response of the nearby slots. ${ }^{29}$ By integrating this approach, many Auto-Tag IDs can be formed as shown in Figure 6. Consequently, a unique tag ID can be generated for each particular tag which will result in avoidance of collisions within the network.

\section{1 | Humidity sensor}

The 27-bit passive chipless RFID tag shows the functionality of humidity sensing when polyamide sheet is integrated into the design.

The prototype is designed by etching the copper radiators on Rogers RT/duroid/5870. The humidity sensing is achieved by placing a DuPont Kapton HN heat resistant conformal tape on the shortest slot and the metal portion corresponding to the respective slot, as shown in Figure 7.

Hence, the last bit obtained due to the shortest slot depicts the sensing bit. The thickness of Kapton HN is 125 $\mu \mathrm{m}$ with a relative permittivity of 3.5 . The resonant frequency of the sensing bit shifts slightly towards the left side 


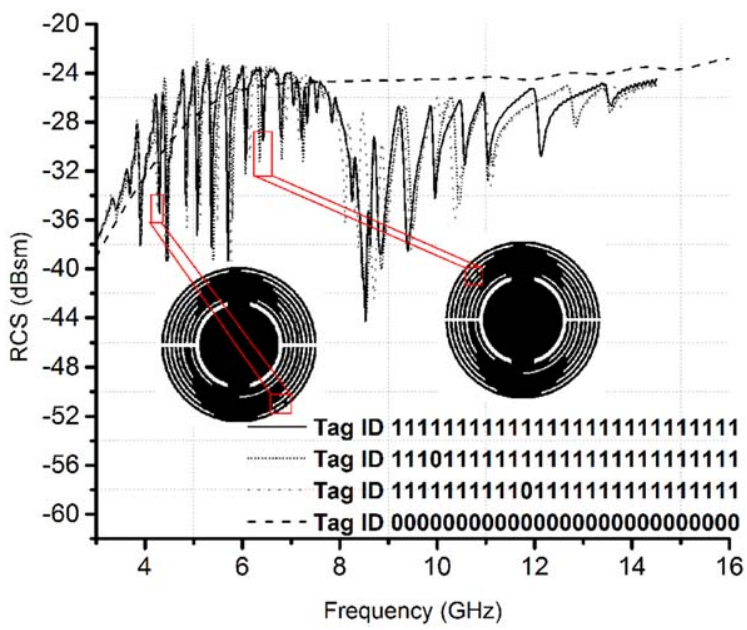

F I G URE 6 RCS response of different tag IDs

with the steady increase in the moisture level. This is because the Kapton HN sheet used for sensing purpose can absorb the moisture. Thus, the novelty of the design lies in the fact that it does not require any external circuit for sensing. Figure 8 shows the RCS feedback with Kapton HN expressed as 'Tag-C'.

The 27-bit chipless humidity sensor tag illustrates a reliable performance. Five prototypes of the proposed tag have been tested. The tag exhibits $0.25 \%$ tolerance in RCS values whereas $0.3 \%$ tolerance is monitored in the required frequency band as shown in Figure 9.

The proposed tag is responsive to moisture present in the environment. It is experimentally analysed using climate chamber by Weiss Technik WK11-180. ${ }^{30}$ The sensing characteristics are scrutinized by implementing diverse levels of moisture in the chamber. The Figure 10 shows that for every $15 \%$ increase in the moisture, the RF response of the respective tag shows a subtle drift towards left side, that is, the lower frequencies.

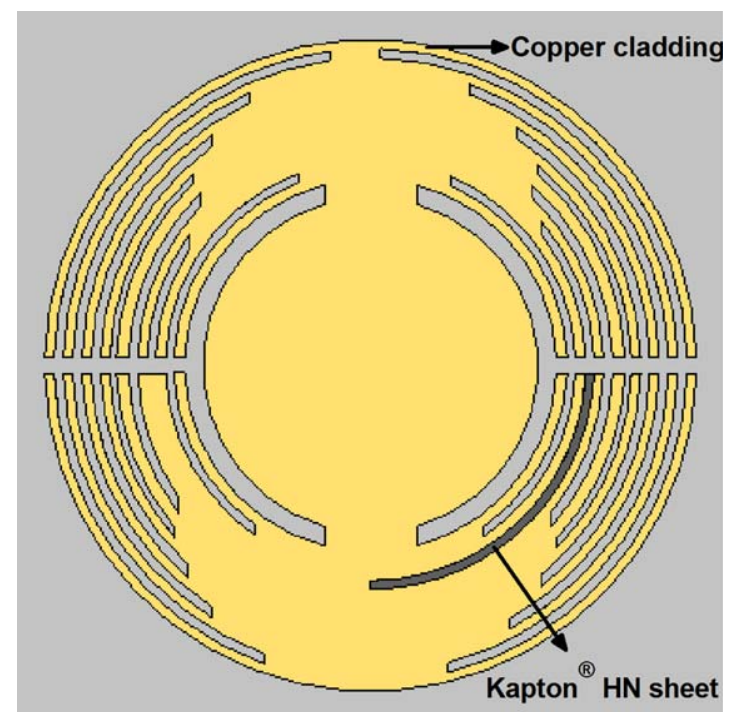

F IG URE 7 Front view of tag design with Kapton HN sheet

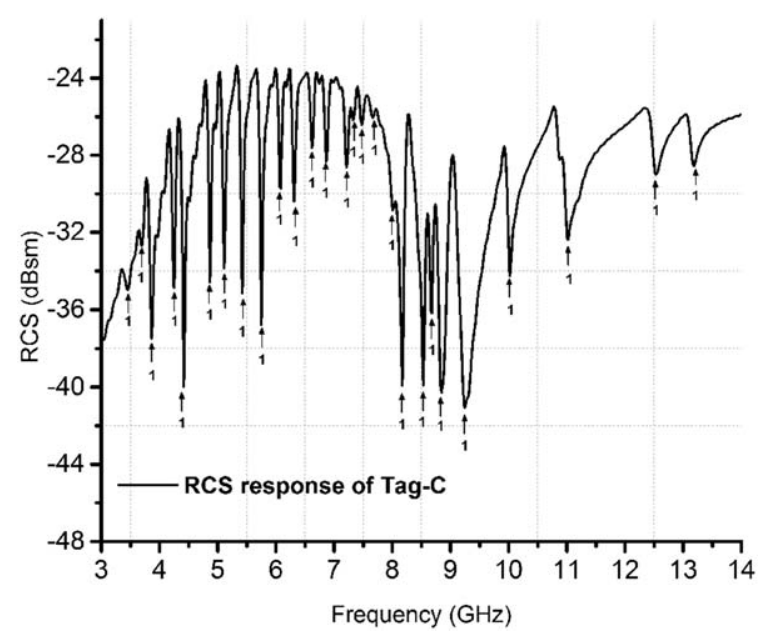

FIG URE 8 RCS response of Tag-C

The change in moisture level of environment ultimately alters the dielectric permittivity of DuPont Kapton HN sheet, deployed on the shortest slot, that is, the sensing slot.

\section{2 | Temperature sensor}

The proposed chipless tag design possesses the functionality of temperature sensing. Temperature sensing can be attained using polymer resins. The dielectric permittivity of these polymer materials alters at high frequencies and are temperature dependant. As the temperature increases, the dielectric properties of polymer resins also increase. Some commonly used polyamide materials are Stanyl, Zenite, Valox, and Zytel.

In the proposed research, temperature sensing is achieved using Stanyl. A linear performance of increasing dielectric permittivity is depicted with temperature. ${ }^{31}$ On increasing the temperature from $25^{\circ} \mathrm{C}$ to $125^{\circ} \mathrm{C}$, the dielectric permittivity of Stanyl polymer changes from 3.5-3.8. The substrate is a fusion of Rogers RT/duroid/5870 and Stanyl polyamide resin as shown in Figure 11. ${ }^{19}$ Stanyl polymer is deployed

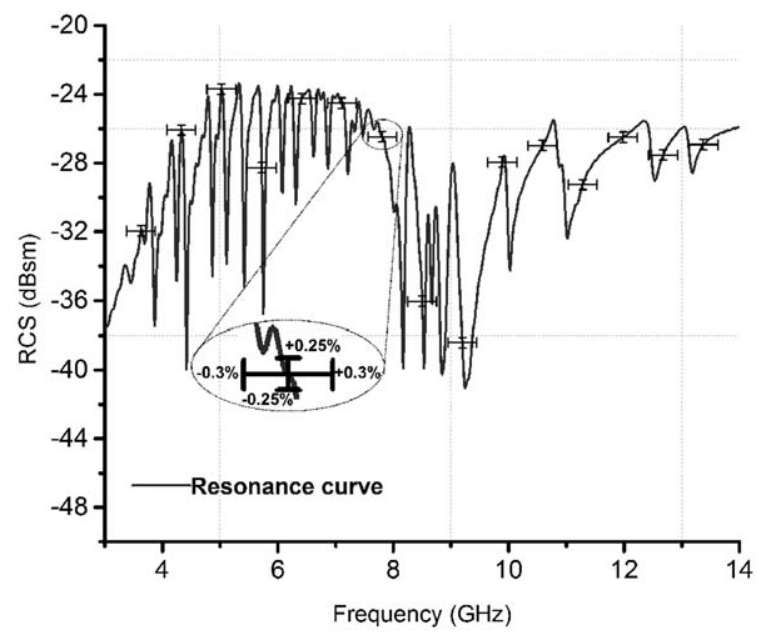

F IGURE 9 Reliability curve of Tag-C 


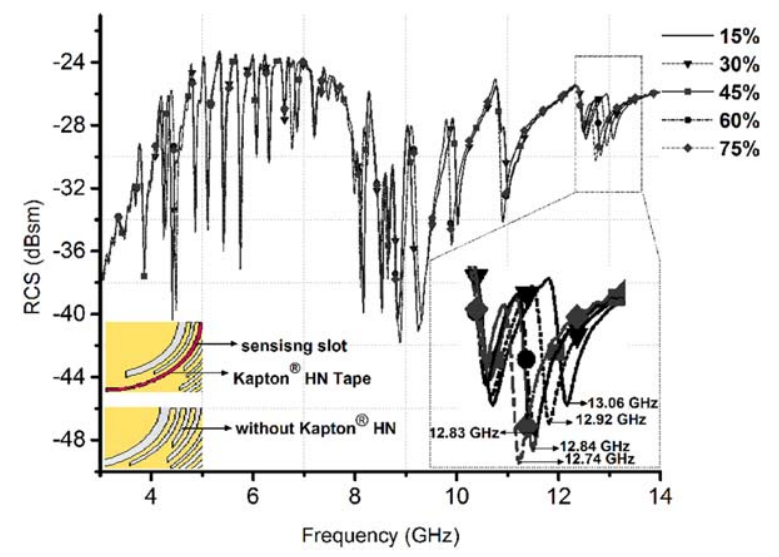

F IG URE 10 Humidity sensing response of the tag

beneath the shortest slot which gives the dip at the highest frequency. This slot is referred as 'sensing slot'.

During the measurement phase, the sensor tag and reader antenna are placed inside environmental chamber namely Weiss Technik WK11-180. ${ }^{30}$ The sensing response of the last bit is shown in Figure 12. The response justifies that with increasing temperature from $25^{\circ} \mathrm{C}$ to $125^{\circ} \mathrm{C}$; the material properties of Stanyl polyamide changes which result in a slight shift of the last bit, that is, the sensing bit, towards lower frequencies. Hence, temperature sensing is achieved using a combination of 2 substrates, that is, Rogers RT/ duroid/5870 and Stanyl polymer.

\section{5 | CONCLUSION}

A finite-sized, data dense, fully passive, chipless RFID tag has been proposed. The tag is excited by a linearly polarized plane wave. This cost-effective tag has the ability to generate $2^{27}$ unique Auto tag IDs. The circular shaped radiators of copper, loaded with slots are analysed for 2 different

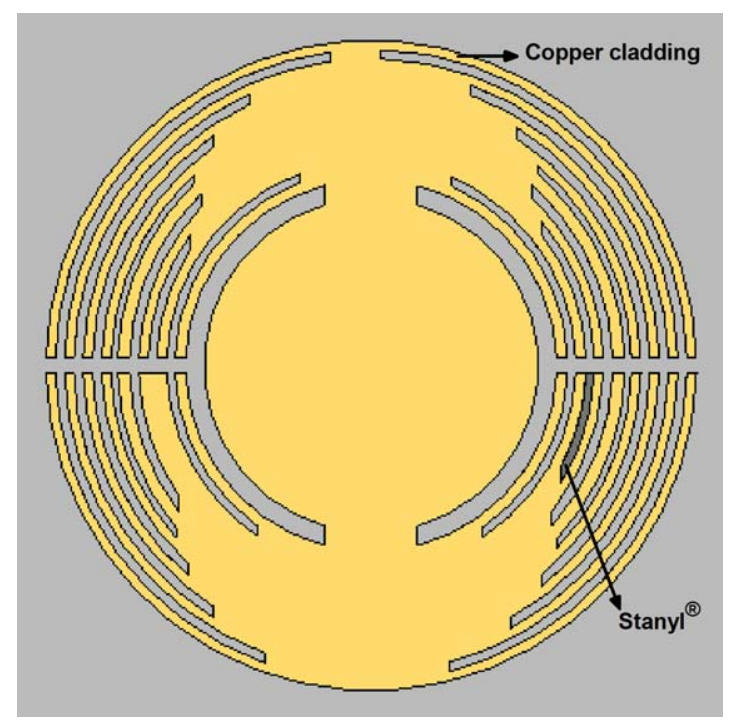

FIGURE 11 Front view of temperature sensor tag

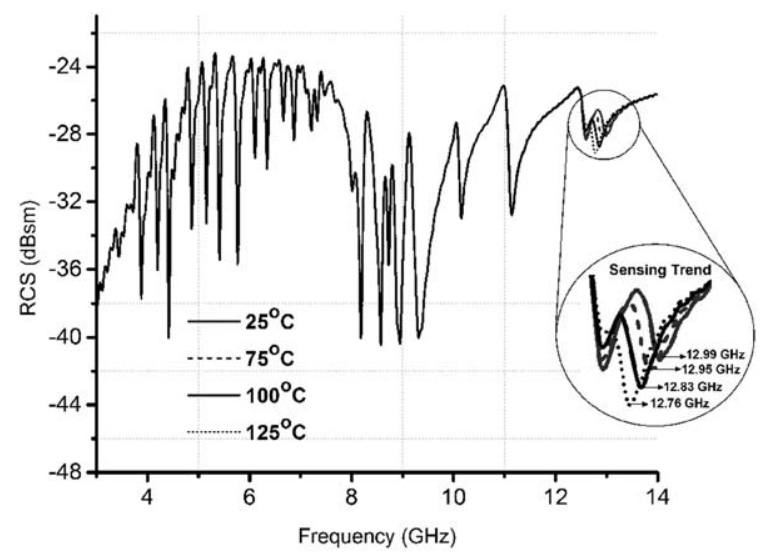

FIGURE 12 Temperature sensing response of the tag

substrates; Rogers RT/duroid/5870 and Rogers RT/duroid/ 5880 flexible within the restricted tag dimension of $23 \times$ $23 \mathrm{~mm}^{2}$. The formulated tag is not only capable of beholding high data capacity but it also offers the additional functionalities of humidity and temperature sensing without requiring any external circuitry. The tag presented in this research article has various advantages which include compactness, robustness, low-cost, sensing, and flexibility, and it can function at a wide range of incident angles. Hence, the temperature and humidity sensor tags can be used in the monitoring and tracking of humidity and temperature sensitive products such as in pharmaceutical industry.

\section{ACKNOWLEDGMENT}

The work was financially supported by Vinnova (The Swedish Governmental Agency for Innovating Systems) and University of Engineering and Technology Taxila, Pakistan through the Vinn Excellence centres program and ACTSENA research group funding, respectively.

\section{REFERENCES}

[1] Al-Fuqaha A, Guizani M, Mohammad M, Aledhari M, Ayyash M. Internet of things: a survey on enabling technologies, protocols, and applications. IEEE Commun Surv Tutor. 2015;17 (4):2347-2376.

[2] Tsai CW, Lai CF, Chiang MC, Yang LT. Data mining for internet of things: a survey. IEEE Commun Surv Tutor. 2014;16(1): 77-97.

[3] Nguyen VT, Nguyen-Thanh N, Yang L, Nguyen DHN, Jabbour C, Murmann B. Cognitive computation and communication: A complement solution to cloud for IoT. Paper presented at: International Conference on Advanced Technologies for Communications (ATC); 2016:222-230; Hanoi, Vietnam.

[4] Ganchev I, Ji Z, O’Droma M. Designing a low-cost data transfer unit for use in IoT applications. Paper presented at: 8th International Congress on Ultra Modern Telecommunications and Control Systems and Workshops (ICUMT); 2016:85-88; Lisbon, Portugal. 
[5] Yang C, Shen W, Wang X. Applications of Internet of Things in manufacturing. Paper presented at: IEEE 20th International Conference on Computer Supported Cooperative Work in Design (CSCWD); 2016:670-675; Nanchang, China.

[6] Amin EM, Bhuiyan MS, Karmakar NC, Winther-Jensen B. Development of a low cost printable chipless RFID humidity sensor. IEEE Sens J. 2014;14(1):140-149.

[7] Polivka M, Havlicek J, Svanda M, Machac J. Improvement in robustness and recognizability of RCS response of U-shaped strip-based chipless RFID tags. IEEE Antennas Wireless Propag Lett. 2016;15:2000-2003.

[8] Sumi M, Dinesh R, Nijas CM, Mridula S, Mohanan P. Frequency coded chipless RFID tag using spurline resonators. Radio Eng J. 2014;24(4):203-208.

[9] Bhuiyan MS, Azad A, Karmakar N. Dual-band modified complementary split ring resonator (MCSRR) based multi-resonator circuit for chipless RFID tag. Paper presented at: IEEE Eighth International Conference on Intelligent Sensors, Sensor Networks and Information Processing; 2013:277-281; Melbourne, VIC.

[10] Havlicek J, Polivka M, Svanda M, Machac J. Capacitively loaded dipoles for chipless RFID transponder. Paper presented at: 26th International Conference Radioelektronika (RADIOELEKTRONIKA); 2016:446-449; Kosice, Slovakia.

[11] Svanda M, Machac J, Polivka M, Havlicek J. A comparison of two ways to reducing the mutual coupling of chipless RFID tag scatterers. Paper presented at: 21st International Conference on Microwave, Radar and Wireless Communications (MIKON); 2016:1-4; Krakow, Poland.

[12] Haraz OM, Ashraf M, Alshebili S, AlShareef MR, Behairy HM. UWB monopole antenna chipless RFID tags using 8-bit open circuit stub resonators. Paper presented at: 21st International Conference on Microwave, Radar and Wireless Communications (MIKON); 2016:1-4; Krakow, Poland.

[13] Shao B, Amin Y, Chen Q, Liu R, Zheng LR. Directly printed packaging-paper-based chipless RFID tag with coplanar resonator. IEEE Antennas Wireless Propag Lett. 2013;12:325-328.

[14] Vena A, Perret E, Tedjni S. A depolarizing chipless RFID tag for robust detection and its FCC compliant UWB reading system. IEEE Trans Microwave Theory Tech. 2013;61(8):2982-2994.

[15] Vena A, Perret E, Tedjini S, et al. Design of chipless RFID tags printed on paper by flexography. IEEE Trans Antennas Propag. 2013;61(12):5868-5877.

[16] Khan MM, Tahir FA, Cheema HM. High capacity polarization sensitive chipless RFID tag. Paper presented at: IEEE International Symposium on Antennas and Propagation \& USNC/URSI National Radio Science Meeting; 2015:1770-1771; Vancouver, BC.

[17] Genovesi S, Coata F, Borgese M, et al. Enhanced chipless RFID tags for sensors design. Paper presented at: 2016 IEEE International Symposium on Antennas and Propagation (APSURSI); 2016:1275-1276; Fajardo, Puerto Rico.

[18] Amin EM, Karmakar N, Winther B. Jensen2, Polyvinyl-Alcohol (PVA)-based RF humidity sensor in microwave frequency. Prog Electromagn Res B. 2013;54:149-166.

[19] Noor T, Habib A, Amin Y, Loo J, Tenhunen H. High-density chipless RFID tag for temperature sensing. Electron Lett. 2016; 52(8):620-622.
[20] Habib A, Amin Y, Azam MA, Loo J, Tenhunen H. Frequency signatured directly printable humidity sensing tag using organic electronics. IEICE Electron Express. 2017;14(3):2016108120161081.

[21] Iqbal MS, Shahid H, Riaz MA, Rauf S, Amin Y, Tenhunen H. FSS inspired polarization insensitive chipless RFID tag. IEICE Electron Express. 2107;14(10):20170243-20170243.

[22] Islam MA, Yap Y, Karmakar NC, Azad A. Compact printable orientation independent chipless RFID tag. Prog Electromagn Res C. 2012;33:55-66.

[23] Martinez M, van der Weide D. Circular polarization on depolarizing chipless RFID tags. Paper presented at: IEEE Radio and Wireless Symposium (RWS); 2016:145-147; Austin, TX.

[24] Habib A, Azam MA, Amin Y, Tenhunen H. Chipless slot resonators for IoT system identification. Paper presented at: IEEE International Conference on Electro Information Technology (EIT); 2016:0341-0344; Grand Forks, ND.

[25] Javed N, Habib A, Akram A, Amin Y, Tenhunen H. 16-bit frequency signature directly printable tag for organic electronics. IEICE Electron Express. 2016;13(11):2016040620160406.

[26] Anam H, Habib A, Jafri SI, Amin Y, Tenhunen H. Directly printable frequency signatured chipless RFID tag for IoT applications. Radio Eng J. 2017;26(1):139-146.

[27] Rauf S, Riaz MA, Shahid H, Iqbal MS, Amin Y, Tenhunen H. Triangular loop resonator based compact chipless RFID tag. IEICE Electron Express. 2017;14(4):2016126220161262.

[28] Vena A, Perret E, Tedjini S. Chipless RFID tag using hybrid coding technique. IEEE Trans Microwave Theory Tech. 2011;59 (12):3356-3364.

[29] Xu L, Huang K. Design of compact trapezoidal bow-tie chipless RFID tag. Int J Antennas Propag. 2015;7:2015.

[30] Feng Y, Xie L, Chen Q, Zheng LR. Low-cost printed chipless RFID humidity sensor tag for intelligent packaging. IEEE Sens J. 2015;15(6):3201-3208.

[31] Gregory TP. Effects of polymer material variation on highfrequency dielectric properties. Paper presented at: The MRS 2009 Spring Meeting; April 2009:1156-D02-05; San Francisco, California. 